\title{
CHD9 upregulates RUNX2 and has a potential role in skeletal evolution
}

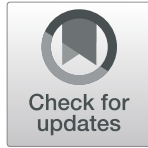

\author{
Axel H. Newton ${ }^{*}$ and Andrew J. Pask
}

\begin{abstract}
Background: Changes in gene regulation are widely recognized as an important driver of adaptive phenotypic evolution. However, the specific molecular mechanisms that underpin such changes are still poorly understood. Chromatin state plays an essential role in gene regulation, by influencing the accessibility of coding loci to the transcriptional machinery. Changes in the function of chromatin remodellers are therefore strong candidates to drive changes in gene expression associated with phenotypic adaptation. Here, we identify amino acid homoplasies in the chromatin remodeller CHD9, shared between the extinct marsupial thylacine and eutherian wolf which show remarkable skull convergence. CHD9 is involved in osteogenesis, though its role in the process is still poorly understood. We examine whether CHD9 is able to regulate the expression of osteogenic target genes and examine the function of a key substitution in the CHD9 DNA binding domain.

Results: We examined whether CHD9 was able to upregulate its osteogenic target genes, RUNX2, Osteocalcin (OC) and ALP in HEK293T cells. We found that overexpression of CHD9 upregulated RUNX2, the master regulator of osteoblast cell fate, but not the downstream genes OC or ALP, supporting the idea that CHD9 regulates osteogenic progenitors rather than terminal osteoblasts. We also found that the evolutionary substitution in the CHD9 DNA binding domain does not alter protein secondary structure, but was able to drive a small but insignificant increase in RUNX2 activation. Finally, CHD9 was unable to activate an episomal RUNX2 promoter-reporter construct, suggesting that CHD9 requires the full chromatin complement for its function.

Conclusions: We provide new evidence to the role of CHD9 in osteogenic differentiation through its newly observed ability to upregulate the expression of RUNX2. Though we were unable to identify significant functional consequences of the evolutionary substitution in HEK293T cells, our study provides important steps forward in the functional investigation of protein homoplasy and its role in developmental processes. Mutations in coding genes may be a mechanism for driving adaptive changes in gene expression, and their validation is essential towards determining the functional consequences of evolutionary homoplasy.
\end{abstract}

Keywords: CReMM, CBFA1, Homoplasy, Convergence, DNA binding, Osteogenesis

\section{Background}

Selection targets traits in response to specific environmental pressures, though the molecular basis of these evolutionary changes remains unclear. Phenotypic development is driven by suites of genes in complex regulatory networks [1-3]. Mutations targeting cis-regulatory regions of key

\footnotetext{
* Correspondence: axel.newton@unimelb.edu.au

School of BioSciences, The University of Melbourne, Melbourne, Victoria, Australia
}

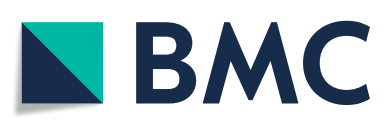

developmental genes are now thought to be the major drivers behind morphological adaptations $[1,4,5]$. However, mutations affecting transcription factors and other protein-coding genes are also known to play critical roles in evolution, particularly through changes in DNA-binding capacity or protein-protein interactions [6, 7]. Recently, studies have examined genome-wide patterns of positive selection and homoplasy in protein coding genes underlying convergent phenotypic traits in mammals. While some key

(c) The Author(s). 2020 Open Access This article is licensed under a Creative Commons Attribution 4.0 International License, which permits use, sharing, adaptation, distribution and reproduction in any medium or format, as long as you give appropriate credit to the original author(s) and the source, provide a link to the Creative Commons licence, and indicate if changes were made. The images or other third party material in this article are included in the article's Creative Commons licence, unless indicated otherwise in a credit line to the material. If material is not included in the article's Creative Commons licence and your intended use is not permitted by statutory regulation or exceeds the permitted use, you will need to obtain permission directly from the copyright holder. To view a copy of this licence, visit http://creativecommons.org/licenses/by/4.0/. The Creative Commons Public Domain Dedication waiver (http://creativecommons.org/publicdomain/zero/1.0/) applies to the data made available in this article, unless otherwise stated in a credit line to the data. 
protein-coding genes implicated in similar phenotypes were recovered, overall adaptive homoplasy was rare [8-10]. Despite this, homoplasy and positive selection has been detected in protein-coding genes with plausible links to the convergent phenotypes examined. However, few studies employ functional analyses to determine their potential contribution to the evolution of phenotypic traits.

The development of complex traits is controlled by the expression of multiple genes in concert [6]. Accordingly, modification of traits is likely driven by small incremental changes to the expression and regulation of this suite of genes [3]. The relationships between gene expression and cis-regulation may be controlled through alterations to epigenetic modifiers (chromatin remodellers), proteins which drive broad changes in gene expression within a specific tissue or cell type [11]. One such mechanism to drive concerted changes in gene expression is through genome-wide alterations in chromatin organization [12, 13]. Chromatin state plays an essential role in gene regulation by altering the accessibility of DNA to transcription factors, repressors, insulators, RNA polymerase and other transcriptional machinery. Mutations in genes that remodel chromatin present strong candidate mechanisms which may drive changes in gene expression underpinning phenotypic adaptation.

CHD (chromodomain helicase DNA-binding) proteins are chromatin remodelling enzymes which reshape the local chromatin architecture to allow access to transcriptional machinery in a tissue-specific context $[11,14]$. CHD proteins facilitate various stages of gene expression, including binding and recruiting transcription factors, histone modifications, and transcriptional elongation, termination and processing [15]. CHD proteins are characterized by two tandem chromodomains and a helicase $\mathrm{C}$ domain, but also possesses additional functional domains which classify them into three subfamilies (reviewed in [14]). Of the nine CHD members, the leaststudied is CHD9. CHD9 is active in mesenchymal stromal stem cells and osteoprogenitor cells, and is suggested to control osteogenic cell fate [16-19]. CHD9 contains a DNA-binding domain which has been shown to associate with $\mathrm{A} / \mathrm{T}$ rich DNA in the promoters of osteogenic genes such as BGLAP (osteocalcin; OC), ALP (alkaline phosphatase) and the master osteogenic regulator RUNX2 (runt-related transcription factor 2) [18-22], though how CHD9 influences the expression of these genes is still unknown. RUNX2 is essential for intramembranous ossification of the facial skeleton [23] and has been implicated as a major driver of craniofacial evolution in mammals, most notably in the Carnivora [24-27].

Interestingly, we identified previously undetected homoplasious amino acids in the CHD9 orthologs of the canids (a family within the Carnivora) and the extinct marsupial thylacine (where it was also found to be under positive selection) [9]. These distantly-related lineages possess remarkable similarities in their skull morphologies $[9,28]$, representing one of the best examples of convergent evolution seen in mammals $[29,30]$. Therefore, evolutionary substitutions in CHD9 present a plausible mechanism behind adaptive changes in craniofacial morphology through modified function. In this study we determine whether overexpression of CHD9 influences the expression of its known osteogenic targets OC, ALP and RUNX2 in vitro; and, examine whether a homoplasious amino acid substitution in the CHD9 DNA-binding domain is able to drive differential gene expression. While adaptive homoplasy is rare [8-10], its occurrence in genes associated with phenotypic traits evokes questions as to how these mutations effect evolutionary gene function. In this study, we provide new evidence into the functional consequences of protein homoplasy, providing insights to the molecular basis of adaptive evolution.

\section{Results}

\section{Phylogenetic distribution of homoplasious amino acids}

We screened alignments of orthologous CHD9 coding sequences to identify shared parallel and convergent amino acid substitutions between the thylacine and canids, with respect to their immediate ancestors. Through this, we identified four individual, homoplasious amino acid substitutions shared between the thylacine and wolf (Fig. 1a). We further examined the evolutionary history of the homoplasious alleles, by investigating their distribution across a simplified vertebrate phylogeny. This revealed that each of the four the amino acid substitutions were not exclusively shared between the thylacine and canids, but rather distributed across additional mammalian lineages (Fig. 1b).

Interestingly, one of the four homoplasious substitutions was found to occur within the DNA-binding domain of CHD9. This substitution at amino acid residue 2384, was found to occur between the thylacine and wolf as a parallel alanine residue (Ala2384). However, additional screening revealed this was in fact the ancestral amino acid among vertebrates. Rather, the last common eutherian ancestor evolved an alanine to threonine substitution (Ala2384Thr), which subsequently reverted back to an alanine residue (Thr2384Ala) in the ancestor of the eutherian Carnivora (including the canids; Fig. 1b). Nevertheless, the location of this amino acid in the DNA binding domain suggests it may possess a functional consequence and provides a tractable candidate to examine the role of evolutionary substitutions.

\section{The Thr2384Ala substitution does not alter protein secondary structure}

Protein coding mutations are often dismissed as major drivers of evolution due to their potential epistatic and 
a)

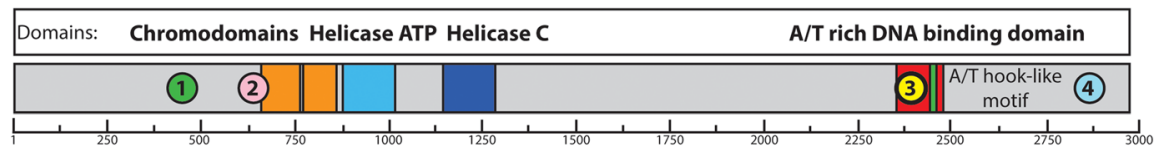

b)

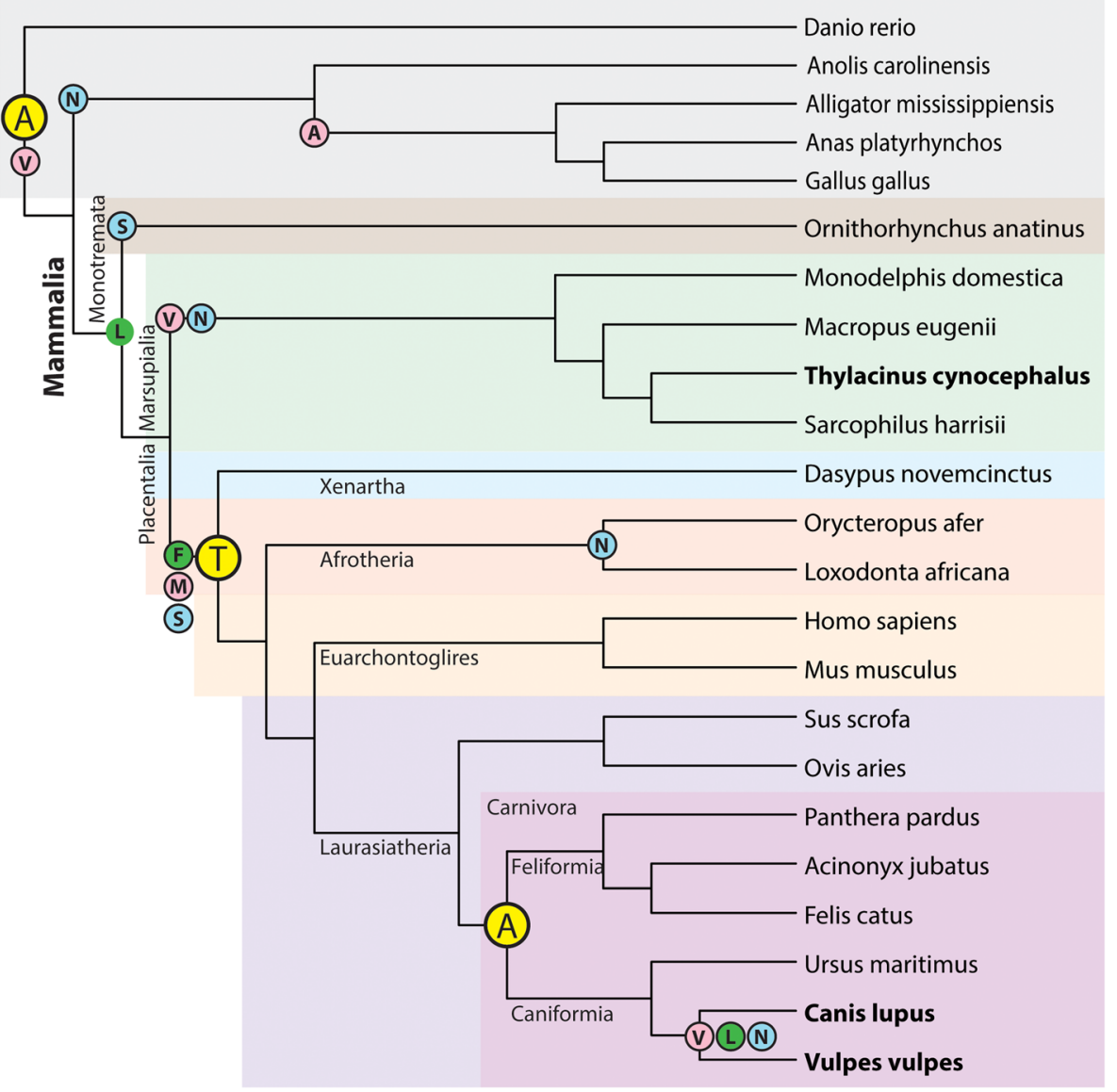

c) (T) A

benign

0.00

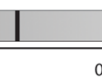

0.20
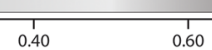
severe

Fig. 1 CHD9 protein and simplified mammalian phylogeny showing the four homoplasious amino acid substitutions. a) Schematic of the CHD9 protein showing functional domains and location of the four identified homoplasious amino acids. b) Simplified mammalian phylogeny showing distribution of each amino acid corresponding to coloured circles in (a). Each amino acid shows different distributions throughout the tree and none were specifically shared between the thylacine and canids (bold text). The Ala2384 homoplasious amino acid identified in the DNA-binding domain (3) is the ancestral vertebrate residue and is highlighted in yellow. c) The Ala2384 homoplasious substitution was predicted to be a benign mutation (score of 0.053; sensitivity: 0.94; specificity: 0.84), predicted by PolyPhen-2 [31]

pleiotropic effects [6]. As such, we determined the impact of the Thr2384Ala amino acid substitution on protein structure, by running the 141 aa CHD9 DNA-binding domain containing the Ala and Thr residue through three protein secondary structure prediction suites, I-TASSER [32], PSIPRED [33] and PolyPhen-2 [31]. Each of these analyses similarly revealed the Thr2384Ala substitution did not alter protein secondary structure and was considered to be a benign change (score of 0.053 ; sensitivity: 0.94; specificity: 0.84; PolyPhen-2) (Fig. 1c). This is in accordance with the role of coding mutations causing subtle changes to protein structure. Instead, the substitution may influence protein function through altered stability, DNA binding affinity, protein transactivation or protein-protein interactions.

\section{Chd9 upregulates RUNX2 expression}

CHD9 has been shown to associate with the promoter of osteogenic genes [20,21], but whether it activates their expression was still unknown. To address this, we examined whether exogenous expression of Chd9 was able to upregulate the endogenous expression of the key osteogenic genes RUNX2, OC and ALP [19, 21]. To detect any potentially subtle effects, we used the non-osteogenic cell line 
HEK293T, as CHD9 is not endogenously active. Exogenous overexpression of Chd9 in HEK293T cells resulted in a 60-fold induction of the Thr2384 allele, and $\sim 40$ induction of the Ala2384 allele over background CHD9 levels (Fig. 2a). Interestingly, we found that overexpression of the Chd9 Thr2384 and Ala2384 allele drove a small but significant $\sim 1.5$-fold increase in endogenous RUNX2 expression compared to the empty vehicle control. However, we found that exogenous $C h d 9$ was unable to upregulate $\mathrm{OC}$ and $A L P$ in vitro (Fig. $2 \mathrm{~b}$ ).

We next determined whether either CHD9 allele drove differential RUNX2 expression. To achieve this, we examined the amount of RUNX2 expression relative to the levels of each Chd9 allele (Thr2384 or Ala2384) in the transfected cells. While both alleles triggered a significant induction of RUNX2 transcription, the two alleles differed minorly in this capacity. Both induced a $\sim 1.5$ fold increase, with a minor, though non-significant, increase by the Ala2384 allele (Fig. 2c).

\section{Chd9 is unable to transactivate the core RUNX2 promoter in HEK293T cells}

CHD9 was sufficient to upregulate the expression of RUNX2 in vitro, but its role in the process was still unclear. Specifically, CHD9 functions to remodel chromatin and alter the accessibility of gene-specific promoters to transcriptional machinery, but whether it additionally acts as a transcription factor to promote gene expression is unknown. To test this, we examined whether exogenous Chd 9 was able to directly transactivate the RUNX2 promoter [35] using an isolated RUNX2 promoterreporter assay out of its regular genomic context. We isolated the $\sim 780 \mathrm{bp}$ core $R U N X 2$ promoter for the thylacine (Thylacinus cynocephalus) and the red fox (Vulpes vulpes, family Canidae) and coupled it to a luciferase reporter. We then compared the two RUNX2 promoter transactivation levels between the Thr2384 and Ala2384 Chd9 alleles.

The thylacine (T.cyn) and red fox (V.vul) RUNX2 promoter-reporter constructs drove low background levels of luciferase activity in HEK293T cells in the absence of exogenous Chd9 (Fig. 3 left, pcDNA vehicle), with slightly elevated activity in the fox (Fig. 3a). Unexpectedly however, we found that neither the CHD9 Thr2384 or Ala2384 allele were able to drive increased transactivation of the thylacine and fox RUNX2 promoter, showing similar activation to background pcDNA vehicle (Fig. 3 middle, right). We did detect a small but significant increase in transactivation of the fox RUNX2 promoter by the Ala2384 allele compared to the Thr2384 allele, but this was not significant compared to the background pcDNA vehicle.

\section{Discussion}

Comparative genomic studies have begun to identify the contributions of coding and non-coding mutations to cases of convergent phenotypic evolution in mammals [8, 10, 36-39], though few have functionally validated these potential evolutionary candidates [7]. Without such analyses, the role these mutations play in adaptive evolution remains unclear. In this study we identified and examined the functional consequence of a homoplasious amino acid substitution in the osteogenic chromatin remodeller CHD9. CHD9 appears to play an important convergent evolutionary role as it has been previously shown to be

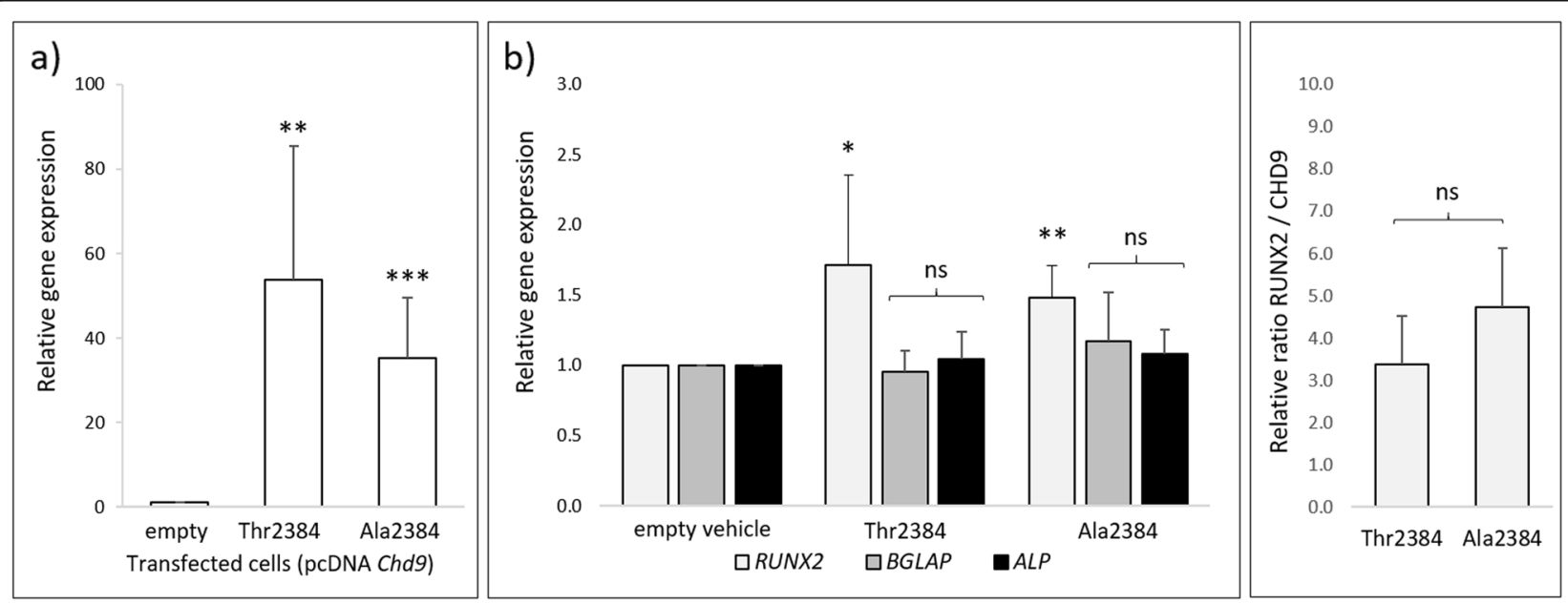

Fig. 2 Chd9 upregulates RUNX2 expression in HEK293T cells. Expression profiles using $\triangle \triangle C T$ analysis [34] of exogenous Chd9 and endogenous downstream osteogenic genes, normalized against HPRT gene expression (HK). a Exogenous CHD9 expression levels. Exogenous Thr2384 and Ala2384 were strongly over-expressed compared to the empty vehicle, with greater expression in the Thr2384 transfected cells. $\mathbf{b}$ Endogenous osteogenic gene expression. Expression of Thr2384 and Ala2384 resulted in a significant increase in endogenous human RUNX2 expression, but not OC or ALP. c Relative RUNX2 expression. RUNX2 was compared against normalized exogenous Chd9 levels to determine the RUNX2 / CHD9 ratio. The Ala2384 allele resulted in a small but non-significant increase in endogenous RUNX2 expression compared to the Thr2384 allele 


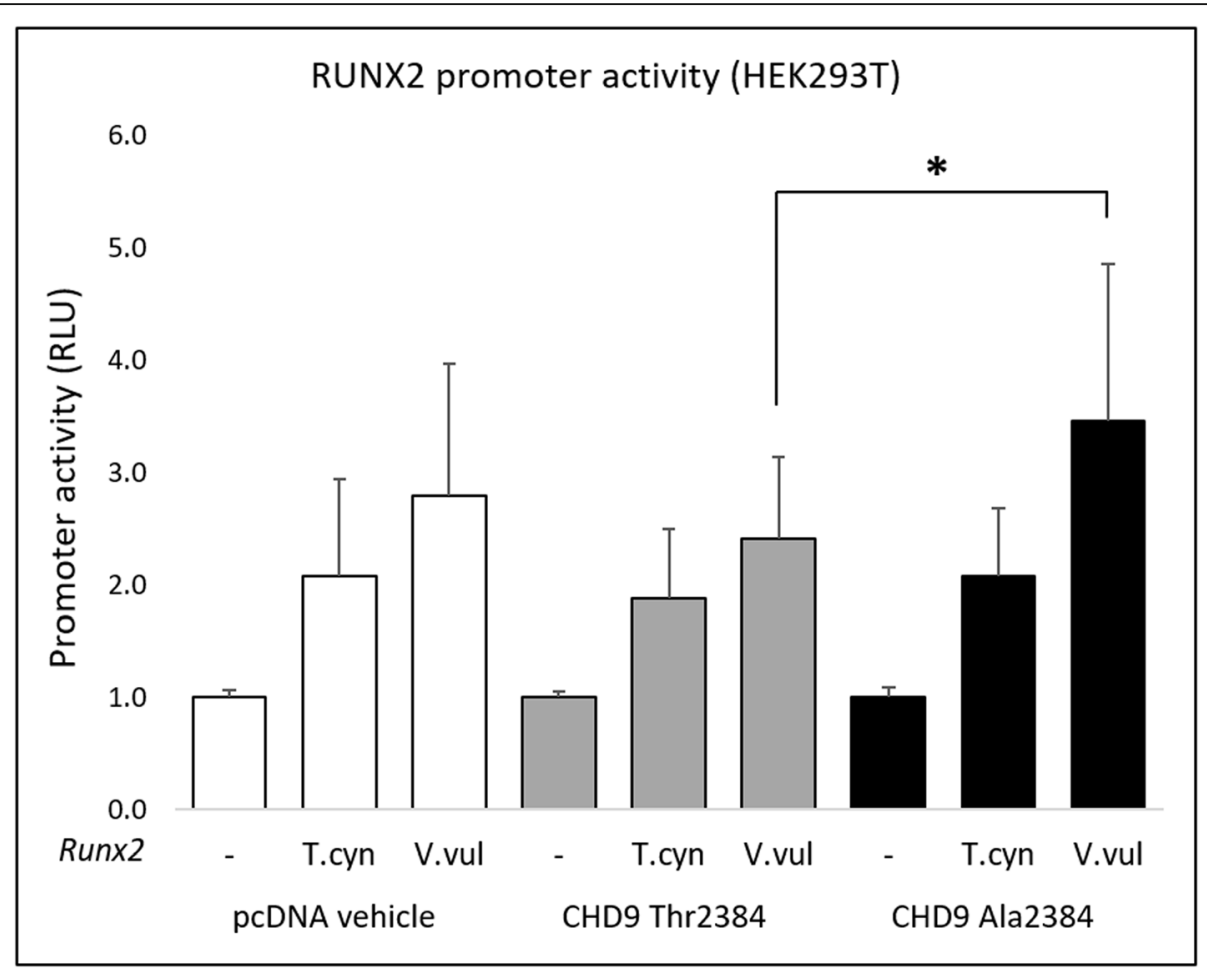

Fig. 3 CHD9 does not transactivate the core RUNX2 promoter in HEK293T cells. Transactivation of the core RUNX2 promoter by the Ala2384 and Thr2384 CHD9 variants in HEK293T cells measured by luciferase activity. Exogenous expression of the CHD9 Thr2384 and Ala2384 allele did not cause differential transactivation of the thylacine (T.cyn) and red fox (V.vul) RUNX2 core promoter compared to the empty vehicle control. The Ala2384 allele drove a small but significant increase in red fox RUNX2 promoter transactivation compared with the Thr2384 allele, though this was not significantly different to the empty vehicle. RLU = relative light units. ${ }^{*}$ denotes significant differences $(P \leq 0.05)$

under positive selection in the thylacine [9], and shares four homoplasious amino acid substitutions with the canids (Fig. 1). Of these substitutions, we identified a key homoplasious alanine residue (Ala2384) in the DNAbinding domain of $C H D 9$ which was found to be benign and not alter protein secondary structure (Fig. 1c). Rather, this substitution causes a minor modification within a functional domain and may instead influence function through modified protein stability, DNA binding affinity, protein transactivation or protein-protein interactions [6]. As such, the occurrence of this substitution provides a tangible system to examine the functional consequence of evolutionary mutations in vitro.

CHD9 is an understudied member of the CHD family of chromatin remodellers $[11,14,15,20]$, but has been suggested to remodel and bind to the promoters of osteogenic genes in mesenchymal stem cells and osteoprogenitors [17-21]. However, the precise biological roles CHD9 play remain relatively unknown, including whether it directly promotes transcription of its osteogenic gene targets. We examined the transactivation potential of $\mathrm{CHD} 9$ through exogenous expression assays in HEK293T cells. HEK293T cells represent a non-osteogenic cell type, but are robust and routinely used for in vitro gene expression assays. Particularly, the lack of endogenous CHD9 or other osteogenic genes allow for detection of potentially subtle interactions in vitro. Here, we found that CHD9 induced significant upregulation of RUNX2, but not the more downstream osteogenic genes $\mathrm{OC}$ and ALP (Fig. 2b). This result confirms that CHD9 not only remodels chromatin $[17,18]$, but also functions to regulate some of its osteogenic gene targets [21]. Given CHD9 was only able to upregulate the expression of the early osteogenic regulator $R U N X 2$, rather than the downstream osteoblast-specific OC [40] or ALP, this suggests that $\mathrm{CHD} 9$ is important in driving the early stages of osteogenesis. This is in accordance with findings that CHD9 is active in the differentiation of osteoprogenitors and mesenchymal stem cells, rather than terminal matrixdepositing osteoblasts [16-19].

With the newly observed role of CHD9 in RUNX2 regulation, we examined whether the homoplasious Ala2384 substitution produces altered RUNX2 expression. We found both alleles were able to increase RUNX2 expression, further confirming CHD9 regulates RUNX2, with the Ala2384 allele driving a small upregulation compared to the Thr2384 allele, however this was not significant (Fig. 2c). Despite the small increase, the lack of significance limits any definitive conclusions as to the whether the amino acid produces functional consequences. However, this result is consistent with the 
theory that phenotypic disparity is driven by multiple small and incremental changes that act in concert $[1,2$, 6]. Additionally, these subtle changes in RUNX2 expression may translate to larger effects in vivo, or when CHD9 is expressed in an osteogenic context. RUNX2 is critical in development of the skeleton [23] and has been implicated in craniofacial evolution within groups of mammals [24-27, 41]. As such, CHD9-mediated alterations to RUNX2 expression and regulation may play a role in differential skeletogenesis between species [41]. However, the larger evolutionary role this substitution may play requires further investigation.

In addition to its known association with osteogenic gene-specific promoters and remodelling capabilities [18, 20, 21], we explored whether CHD9 possesses an additional role as a transcription factor, able to directly transactivate $R U N X 2$ through its core promoter [42]. We found that CHD9 was unable to activate the isolated RUNX2 promoter (Fig. 3), suggesting this might not be the case. However, the lack of activity could also be explained by the exogenous conditions of the system for a few reasons. Firstly, the $\geq 750$ bp core $R U N X 2$ promoter is a 'bone-specific element' active only in osteoblasts [35]. In contrast, the complete endogenous $R U N X 2$ promoter has been described as a $3 \mathrm{~kb}$ element which contains multiple upstream tissue-specific response elements $[35,43]$ which may be required to facilitate the CHD9-induced activation we observed. Secondly, CHD9 regulatory activity may require an endogenous chromatin environment, since this gene acts as a chromatin remodeller. Thus, it may not be able to function in an extra-chromosomal (episomal) context with a transfected RUNX2 promoter. CHD9 function has been tightly associated with active histone modifications at promoters [14, 17, 18]. As such, CHD9 may require chromatin-bound transcriptional co-factors to activate its target promoters and gene transcription. Finally, a more biologically relevant role of CHD9 during osteogenesis may be resolved through the use of other in vitro models such as mesenchymal stem cells [20] or osteoprogenitors cells [19] where CHD9 and RUNX2 are both active. Nevertheless, our findings provide new evidence that CHD9 regulates RUNX2 expression, further implicating it in osteogenesis [16, 20, 21].

\section{Conclusion}

CHD9 is a chromatin remodeller and epigenetic modifier suggested to be active in osteogenesis and skeletal development $[16,19-21,44]$, though the precise roles it plays throughout these processes are still poorly understood. Our data provide a novel new role for CHD9 in osteogenesis though its ability to regulate RUNX2 expression. Additionally, our examinations of CHD9 homoplasy revealed a candidate amino acid substitution in the CHD9 DNA binding domain prompting functional validation. While we saw a small increase in RUNX2 activation by the homoplasious allele, this was not significant. Homoplasious amino acid substitutions in coding genes are generally rare and not enriched between convergent traits [8-10], though their occasional presence requires experimental validation. In this study, we take necessary steps investigating the functional roles of protein homoplasy but highlight that these effects may be subtle and difficult to identify. The presence of additional untested homoplasies in CHD9 also evoke interesting questions as to how these substitutions may function in concert. Nevertheless, our newly observed ability for CHD9 to regulate RUNX2, combined with its potential to differentially activate its expression, provides an attractive mechanism by which adaptive phenotypic evolution may be controlled at the molecular level.

\section{Methods}

\section{Identification of gene homoplasy}

The method for identifying thylacine-canid amino acid homoplasies has been previously described [9]. Briefly, CHD9 Sequences from the thylacine and canids (wolf, coyote, jackal, red fox and arctic fox) were extracted from previously published reference-based genome assemblies. High-confidence 1:1 orthologous CHD9 sequences from a variety of other mammals (Tasmanian devil, wallaby, opossum, elephant, human, bat, sheep, horse, ferret and dog) were downloaded from Ensembl 84 BioMart. Codon alignments of all sequences were then produced using the translation-aware aligner MACSE version $1.01 \mathrm{~b}$ with default parameters. Published phylogenies for all included species were assumed [45] and ancestral sequence reconstructions were predicted for all internal nodes using CodeML (PAMLv4.7). Each alignment column was then examined for amino acids shared between the thylacine and canids, but which differed from that of their ancestors or living relatives. Previously, all homoplasious acids were defined as residues shared between the thylacine and canids, but which differed with respect to their immediate ancestors [9]. Through this approach we identified new thylacine and canid protein coding homoplasies to those previously reported [9] due to the strict focus on convergent and parallel amino acid changes. Cases where the ancestral states were the same were defined as parallel, while cases where their ancestral amino acids differed were defined as convergent.

\section{Phylogenetic distribution and structural properties of the substitution}

The use of high confidence 1:1 mammalian orthologs to detect CHD9 homoplasy limited our number of species in our screen. We therefore examined the distribution of 
the 2384 amino acid residue across a broader vertebrate phylogeny. CHD9 coding sequences from a wide range of vertebrate species were extracted from GenBank and aligned. We then constructed a simplified vertebrate phylogenetic tree using PhyloT (biobyte solutions, $\mathrm{GmbH}$ ) based on NCBI taxonomy, to visualize the specific amino acid for each linage throughout the branches (Fig. 1b).

To determine whether the homoplasious amino acid altered the physical properties of CHD9, we investigated protein folding and secondary structure through computational tools. The 141 amino acid DNA binding domain containing both the Thr2384 and Ala2384 amino acid motif was input into PSIPRED [33] and I-TASSER [32] to determine secondary structure of the domain. In parallel, we input the full length CHD9 CDS into PolyPhen2 [31] to predict the impact of the substitution on CHD9 structure and function.

\section{CHD9 cloning}

All functional experiments were performed using the mouse Chd9 CDS, which natively contains the Thr2384 allele. To generate the Chd 9 coding sequence, Chd9 mRNA was isolated from MC3T3-E1 cells and converted to cDNA, serving as the amplification template. To obtain the full length Chd9 CDS, two separate PCR reactions using Phusion HSII High Fidelity polymerase (Invitrogen) were performed on the $5^{\prime}(4.5 \mathrm{~kb})$ and $3^{\prime}(4.7 \mathrm{~kb})$ ends of the transcript to obtain two $600 \mathrm{bp}$ overlapping amplicons. A second round of PCR was performed using outer primers to obtain the full-length $(8.65 \mathrm{~kb})$ product including the Thr2384 residue. Correct sequence identify was confirmed by capillary electrophoresis sequencing (Centre for Translational Pathology, University of Melbourne) using 12 unique tiling primers. The full-length mouse Chd9 CDS was subcloned into the pGEM-T easy vector (Promega) for propagation and purification. For primer sequences see Supplementary Table 1.

We next generated the Ala2384 amino acid allele. To do this we designed and synthesized a 490 bp gBlock (Integrated DNA Technologies, Iowa, USA), containing a Thr $>$ Ala codon substitution at the 2384 residue. The gBlock additionally contained two unique flanking restriction enzyme sites - BbeI (5') and SfoI (3') (NEB). The pGEM_Chd9Thr2384 plasmid was linearized with BbeI and SfoI to remove the Thr2384 codon and then purified (QIAQuick Gel Extraction kit, QIAGEN). The gBlock containing the Ala2384 residue was digested with BbeI and SfoI and ligated into the linearized pGEMChd9 to obtain the full length CDS with Ala2384 amino acid pGEM_Chd9Ala2384. The full length Ala2384 product was sequenced to confirm the presence of the Ala2384 substitution.
The pGEM_Chd9Thr2384 and pGEM_Chd9Ala2384 plasmids were further digested with NotI (NEB) to excise the full-length CDS from the vector backbone. The resulting full-length products were ligated and subcloned into the pcDNA3.1(+) Mammalian Expression Vector (ThermoFisher) for downstream experimental analyses.

\section{RUNX2 promoter-reporter construct design}

We generated RUNX2 promoter-reporter constructs from the thylacine (Thylacinus cynocephalus) and canid the red fox (Vulpes vulpes) [9]. The core RUNX2 promoter has been previously described as a $\sim 960$ bp element in mouse and $\geq 750 \mathrm{bp}$ element in other mammals [35]. The thylacine promoter was extracted from the thylacine referenced genome assembly using the Tasmanian devil sequence [9]. This was aligned against other placental and marsupial sequences to determine the core marsupial / placental RUNX2 promoter. We determined the promoter as a $\sim 780 \mathrm{bp}$ element in the thylacine and $\sim 800 \mathrm{bp}$ element in the red fox, which despite their large evolutionary divergence are highly conserved. The thylacine $780 \mathrm{bp}$ element was synthesized as a single gBlock (Integrated DNA Technologies, Iowa, USA), while the fox element was amplified from red fox gDNA with canid specific primers (Supplementary Table 1). Both products contained flanking NheI and HindIII restriction sites for subcloning. The thylacine and canid RUNX2 promoter constructs were digested and ligated into the luciferase reporter pGL4.10[luc2] (Promega).

\section{Cell culture and transfection}

Human embryonic kidney (HEK293T) cells were maintained in DMEM (ThermoFisher) media containing 10\% FBS. The media was replaced every $2-3$ days. Cells were passaged at $90 \%$ confluency. HEK293T cells were seeded in 6 well plates and transfected using lipofectamine 3000 (ThermoFisher) according to the manufacturer's instructions. Cells were either transfected with $2.5 \mu \mathrm{g}$ of empty vector (pcDNA), or plasmids containing the Thr2384 or Ala2384 alleles and incubated for $24 \mathrm{~h}$. Each transfection experiment was repeated 5 times for reproducibility.

\section{RNA extraction, gene expression and quantification}

Transfected cells were harvested, and RNA was extracted using the GenElute Mammalian Total RNA Miniprep Kit (Sigma Aldrich). RNA was treated with Turbo DNA-free DNase (Ambion) to remove all residual traces of gDNA. The resulting RNA was stored at $80^{\circ} \mathrm{C}$. Total RNA was converted to cDNA using the SuperScript $^{\text {tix }}$ III First-Strand Synthesis System (ThermoFisher). RT-qPCR was performed to examine expression levels for each of the genes of interest in cells transfected with empty vehicle, or Chd9 possessing the Thr2384 and 
Ala2384 alleles $(n=5)$. Thr2384 or Ala2384 transfected cell gene expression values were normalized against the housekeeping gene $H P R T$ and the un-transfected vehicle using the $\triangle \triangle C T$ method [34]. As exogenous levels of Thr2384 and Ala2384 CHD9 were not equal in our transfection replicates, we additionally compared normalized expression levels of $R U N X 2$ directly against the normalized exogenous levels of the Thr2384 and Ala2384 CHD9 alleles and determined their levels as a ratio. Primer sequences are listed in Supplementary Table 1.

\section{Luciferase promoter-reporter assays}

HEK293T cells were seeded in white flat-bottom 96-well plates and allowed to recover for $24 \mathrm{~h}$. The following day, cells were transfected using Lipofectamine 3000, according to manufacturer instructions, with either empty pcDNA, Thr2384 or Ala2384; as well as either the empty pGL4.10 vector, thylacine or fox RUNX2 promoterluciferase reporter. $R U N X 2$ promoter driven firefly luciferase and Renilla luciferase activity were analysed $48 \mathrm{~h}$ later using the Dual-Luciferase Reporter Assay System (Promega) on a FLUOstar OPTIMA dual plate reader (BMG Labtech Ortenberg, Germany). Firefly luciferase values were normalized against Renilla luciferase to obtain relative promoter activity, measured as relative light units (RLU). Each transfection and luciferase assay were repeated 5 times for reproducibility.

\section{Supplementary information}

Supplementary information accompanies this paper at https://doi.org/10. 1186/s12860-020-00270-5.

Additional file 1. Supplementary Table 1 - Primer sequences used in the study.

\section{Abbreviations}

CHD9: Chromatin helicase DNA-binding protein 9; RUNX2: Runt-related transcription factor 2; RT: Reverse transcription; PCR: Polymerase chain reaction

\section{Acknowledgments}

We thank Pascal Bernard for advice and support on experimental design and methodology used throughout the project. Additionally, we thank Charles Feigin for assistance accessing thylacine sequence data and bioinformatic analyses.

\section{Authors' contributions}

Both authors conceived and designed the project. AHN performed all experiments, collected and analysed the data. AHN and AP wrote the manuscript and approved the final version.

\section{Funding}

The design of the study, collection, analysis, and interpretation of data was supported by an Australian Research Council Future Fellowship FT140100964 to AJP. The writing of the manuscript was supported by an Australian Postgraduate Award and The Albert Shimmins Fund writing-up award to AHN.

\section{Availability of data and materials}

All data generated or analysed during this study are included in this published article [and its supplementary information files].

Ethics approval and consent to participate

All protocols were approved by The University of Melbourne Ethics Committees.

Consent for publication

Not applicable.

\section{Competing interests}

The authors declare that they have no competing interests.

Received: 29 July 2019 Accepted: 31 March 2020

Published online: 15 April 2020

\section{References}

1. Carroll SB. Endless forms: the evolution of gene regulation and morphological diversity. Cell. 2000;101:577-80. https://doi.org/10.1016/ s0092-8674(00)80868-5.

2. Carroll SB. Evolution at two levels: on genes and form. PLoS Biol. 2005;3: 1159-66.

3. Cretekos CJ, Wang Y, Green ED, Martin JF, Rasweiler JJ IV, Behringer RR, et al. Regulatory divergence modifies limb length between mammals. Genes Dev. 2008;22:141-51. https://doi.org/10.1101/gad.1620408.

4. Kvon EZ, Kamneva OK, Melo US, Barozzi I, Osterwalder M, Mannion BJ, et al. Progressive Loss of Function in a Limb Enhancer during Snake Evolution. Cell. 2016;167:633-642.e11.

5. Shapiro MD, Bell MA, Kingsley DM. Parallel genetic origins of pelvic reduction in vertebrates. Proc Natl Acad Sci. 2006;103:13753-8. https://doi. org/10.1073/pnas.0604706103.

6. Lynch VJ, Wagner GP. Resurrecting the role of transcription factor change in developmental evolution. Evolution. 2008;62:2131-54

7. Powder KE, Cousin H, McLinden GP, Craig AR. A nonsynonymous mutation in the transcriptional regulator lbh is associated with cichlid craniofacial adaptation and neural crest cell development. Mol Biol Evol. 2014;31:311324.

8. Foote AD, Liu Y, Thomas GWCC, Vinař T, Alföldi J, Deng J, et al. Convergent evolution of the genomes of marine mammals. Nat Genet. 2015;47:272-5. https://doi.org/10.1038/ng.3198.

9. Feigin CY, Newton AH, Doronina L, Schmitz J, Hipsley CA, Mitchell KJ, et al. Genome of the Tasmanian tiger provides insights into the evolution and demography of an extinct marsupial carnivore. Nat Ecol Evol. 2018;2:182-92. https://doi.org/10.1038/s41559-017-0417-y.

10. Parker J, Tsagkogeorga G, Cotton JA, Liu Y, Provero P, Stupka E, et al. Genome-wide signatures of convergent evolution in echolocating mammals. Nature. 2013;502:228-31. https://doi.org/10.1038/nature12511.

11. Micucci JA, Sperry ED, Martin DM. Chromodomain helicase DNA-binding proteins in stem cells and human developmental diseases. Stem Cells Dev. 2015;24:917-26. https://doi.org/10.1089/scd.2014.0544.

12. Clapier CR, Iwasa J, Cairns BR, Peterson CL. Mechanisms of action and regulation of ATP-dependent chromatin-remodelling complexes. Nat Rev Mol Cell Biol. 2017:18:407-22. https://doi.org/10.1038/nrm.2017.26.

13. Clapier CR, Cairns BR. The biology of chromatin remodeling complexes. Annu Rev Biochem. 2009:78:273-304. https://doi.org/10.1146/annurev. biochem.77.062706.153223

14. Marfella CGA, Imbalzano AN. The Chd family of chromatin remodelers. Mutat Res. 2007;618:30-40

15. Murawska M, Brehm A. CHD chromatin remodelers and the transcription cycle. Transcription. 2011;2:244-53.

16. Shur I, Benayahu D. Characterization and functional analysis of CReMM, a novel chromodomain helicase DNA-binding protein. J Mol Biol. 2005;352: 646-55.

17. De Dieuleveult M, Yen K, Hmitou I, Depaux A, Boussouar F, Dargham DB, et al. Genome-wide nucleosome specificity and function of chromatin remodellers in ES cells. Nature. 2016:530:113-6. https://doi.org/10.1038/ nature16505.

18. Salomon-Kent R, Marom R, John S, Dundr M, Schiltz LR, Gutierrez J, et al. New face for chromatin-related Mesenchymal modulator: n-CHD9 localizes 
to nucleoli and interacts with ribosomal genes. J Cell Physiol. 2015;230: 2270-80.

19. Marom R, Shur I, Hager GL, Benayahu D. Expression and regulation of CReMM, a chromodomain helicase-DNA-binding (CHD), in marrow stroma derived osteoprogenitors. J Cell Physiol. 2006;207:628-35.

20. Shur I, Solomon R, Benayahu D. Dynamic interactions of chromatin-related Mesenchymal modulator, a Chromodomain helicase-DNA-binding protein with promoters in Osteoprogenitors. Stem Cells. 2006;24:1288-93. https:// doi.org/10.1634/stemcells.2005-0300.

21. Shur I, Socher R, Benayahu D. In vivo association of CReMM/CHD9 with promoters in osteogenic cells. J Cell Physiol. 2006;207:374-8.

22. Komori T, Yagi H, Nomura S, Yamaguchi A, Sasaki K, Deguchi K, et al. Targeted disruption of $\mathrm{Cbfa}$ results in a complete lack of bone formation owing to maturational arrest of osteoblasts. Cell. 1997;89:755-64. https://doi. org/10.1016/50092-8674(00)80258-5.

23. James MJ, Järvinen E, Wang XP, Thesleff I. Different roles of Runx2 during early neural crest-derived bone and tooth development. J Bone Miner Res. 2006;21:1034-44.

24. Sears KE, Goswami A, Flynn JJ, Niswander LA. The correlated evolution of Runx2 tandem repeats, transcriptional activity, and facial length in Carnivora. Evol Dev. 2007:9:555-65.

25. Ritzman TB, Banovich N, Buss KP, Guida J, Rubel MA, Pinney J, et al. Facing the facts: the Runx2 gene is associated with variation in facial morphology in primates. J Hum Evol. 2017;111:139-51. https://doi.org/10.1016/j.jhevol. 2017.06.014.

26. Pointer MA, Kamilar JM, Warmuth V, Chester SGB, Delsuc F, Mundy NI, et al. RUNX2 tandem repeats and the evolution of facial length in placental mammals. BMC Evol Biol. 2012;12:103.

27. Ferraz T, Rossoni DM, Althoff SL, Pissinatti A, Paixão-Cortês VR, Bortolini MC, et al. Contrasting patterns of RUNX2 repeat variations are associated with palate shape in phyllostomid bats and New World primates. Sci Rep. 2018;8: $1-10$.

28. Newton AH, Spoutil F, Prochazka J, Black JR, Medlock K, Paddle RN, et al. Letting the 'cat' out of the bag: pouch young development of the extinct tasmanian tiger revealed by X-ray computed tomography. R Soc Open Sci. 2018:5:171914. https://doi.org/10.1098/rsos.171914.

29. Werdelin L. Comparison of skull shape in marsupial and placental carnivores. Aust J Zool. 1986:34:109-17.

30. Goswami A, Milne N, Wroe S. Biting through constraints: cranial morphology, disparity and convergence across living and fossil carnivorous mammals. Proc R Soc B Biol Sci. 2011;278:1831-9. https://doi.org/10.1098/ rspb.2010.2031.

31. Meyer CA, Liu XS. Identifying and mitigating bias in next-generation sequencing methods for chromatin biology. Nat Rev Genet. 2014;15:709-21. https://doi.org/10.1038/nrg3788.

32. Yang J, Yan R, Roy A, Xu D, Poisson J, Zhang Y. The I-TASSER suite: protein structure and function prediction. Nat Methods. 2014;12:7-8. https://doi.org/ 10.1038/nmeth.3213.

33. Jones DT. Protein secondary structure prediction based on position-specific scoring matrices. J Mol Biol. 1999;292:195-202. https://doi.org/10.1006/jmbi. 1999.3091.

34. Wojciechowska-Durczyńska K, Krawczyk-Rusiecka K, Cyniak-Magierska A, Zygmunt A, Gałecka E, Lewiński A. Relative quantification of PIK3CA gene expression level in fine-needle aspiration biopsy thyroid specimens collected from patients with papillary thyroid carcinoma and non-toxic goitre by real-time RT-PCR. In: Dorak T, editor. Thyroid Research. 1st ed. Newcastle: Garland Science; 2010. p. 5.

35. Tai PWL, Wu H, Gordon JAR, Whitfield TW, Barutcu AR, van Wijnen AJ, et al. Epigenetic landscape during osteoblastogenesis defines a differentiationdependent Runx2 promoter region. Gene. 2014;550:1-9.

36. Zhang G, Li C, Li Q, Li B, Larkin DM, Lee C, et al. Comparative genomics reveals insights into avian genome evolution and adaptation. Science. 2014; 346:1311-20.

37. Li Y, Liu Z, Shi P, Zhang J. The hearing gene Prestin unites echolocating bats and whales. Curr Biol. 2010;20:55-6.

38. Davies KTJ, Tsagkogeorga G, Rossiter SJ. Divergent evolutionary rates in vertebrate and mammalian specific conserved non-coding elements (CNES) in echolocating mammals. BMC Evol Biol. 2014;14:1-19.

39. Sackton TB, Grayson P, Cloutier A, Hu Z, Liu JS, Wheeler NE, Gardner PP, Clarke JA, Baker AJ, Clamp M, Edwards SV. Convergent regulatory evolution and loss of flight in paleognathous birds. Science. 2019;364:74-8.
40. Hauschka PV, Lian JB, Cole DE, Gundberg CM. Osteocalcin and matrix Gla protein: vitamin K-dependent proteins in bone. Physiol Rev. 2017:69:9901047. https://doi.org/10.1152/physrev.1989.69.3.990.

41. Newton AH, Feigin CY, Pask AJ. RUNX2 repeat variation does not drive craniofacial diversity in marsupials. BMC Evol Biol. 2017;17:1-9.

42. Tai PWL, Wu H, Van Wijnen AJ, Stein GS, Stein JL, Lian JB. Genome-wide DNase hypersensitivity, and occupancy of RUNX2 and CTCF reveal a highly dynamic gene regulome during MC3T3 pre-osteoblast differentiation. PLOS One. 2017;12:1-23.

43. Xiao ZS, Liu SG, Hinson TK, Quarles LD. Characterization of the upstream mouse Cbfa1/Runx2 promoter. J Cell Biochem. 2001:82:647-59.

44. Manning BJ, Yusufzai T. The ATP-dependent chromatin remodeling enzymes CHD6, CHD7, and CHD8 exhibit distinct nucleosome binding and remodeling activities. J Biol Chem. 2017;292:11927-36.

45. Bininda-Emonds ORP, Cardillo M, Jones KE, MacPhee RDE, Beck RMD, Grenyer R, et al. The delayed rise of present-day mammals. Nature. 2007; 446:507-12. https://doi.org/10.1038/nature05634.

\section{Publisher's Note}

Springer Nature remains neutral with regard to jurisdictional claims in published maps and institutional affiliations.

\section{Ready to submit your research? Choose BMC and benefit from:}

- fast, convenient online submission

- thorough peer review by experienced researchers in your field

- rapid publication on acceptance

- support for research data, including large and complex data types

- gold Open Access which fosters wider collaboration and increased citations

- maximum visibility for your research: over $100 \mathrm{M}$ website views per year

At BMC, research is always in progress.

Learn more biomedcentral.com/submissions 